Integrating digital humanities into the web of scholarship with SHARE:

An exploration of requirements

\title{
Enhancing the Humanities
}

Contemporary scholarship is interdisciplinary, multimodal and distributed across a wide network of tools, repositories, and websites (Maron, 2014). A digital humanities (DH) project may produce more than one manuscript (book or articles), each published on a different publisher's website, grant award information, any number of preprints on MLA CORE or other services, data sets and code books on Dryad or Figshare, and text mining or cleaning scripts on github. By linking these dispersed research objects with one another, they can be evaluated and understood as part of the same intellectual work, thus increasing our understanding of the scholarship and limiting any intellectual stratification in the community of scholars based on their different contributions (collections of primary sources, computational research, and tools, e.g.). On the other hand, when

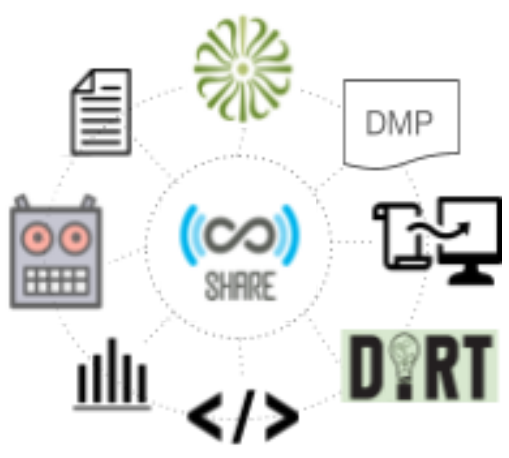
individual project components such as scripts are too tightly bundled and isolated on project websites, they are hidden from networked search and discovery tools, resulting in similar problems.

SHARE - a partnership between the Association of Research Libraries (ARL) and the Center for Open Science (COS) - is part of a growing open academic infrastructure movement. SHARE's open source software aggregates free, open metadata about scholarship and links scholarly activity across the research lifecycle and in any discipline. By working closely with scholars and librarians in the DH community, SHARE can help to resolve some of these issues, by offering a means for scholars to link all the components of their work; for librarians to have a means to accurately track usage of all the components of a DH project; for scholars and students to quickly find the relevant scholarship and primary sources they need; and for new project leaders to quickly gain an understanding of all the existing content and tools at their disposal. Aggregating information about DH projects and components will also assist campus units in optimizing their resources and services in support of this scholarship.

Built as a public good, SHARE supports the project lifecycle and enables the long-term stewardship of all forms of research activity by making that research discoverable. Scholarly repositories or websites may or may not be administered by libraries, but collectively their contents represent collections of abiding interest to the research library and scholarly community. Academic institutions and organizations have missions to support, preserve, and assist in the discovery of scholarly materials created locally. As a community-built resource, SHARE's proposed engagement with DH practitioners can serve as a framework for libraries to consider whether and how to house digital humanities project outputs in their repositories, and/or to reflect metadata about those projects and provide links to them. Part of SHARE's core value proposition is that by aggregating and linking metadata, open repositories need not compete with one another for content, enabling content creators to choose platforms that best suit their individual or collaborative workflow needs. Content stewards, by the same token, can make informed (even coordinated) decisions and plans for preservation of resulting digital assets.

SHARE was initially created in response to a federal directive to make scientific results more publicly available (Holdren, 2013). However, its API and metadata schema are flexible and discipline-agnostic, and SHARE's data providers (institutional and disciplinary repositories) contain multidisciplinary research outputs. At SHARE's 2016 Community Meeting, Kathleen Fitzpatrick-Associate Executive Director and Director of Scholarly Communication at the Modern Language Association-presented on 
the MLA Commons and emerging Humanities Core initiative to great enthusiasm from library-administered institutional repository leaders, who saw in SHARE an opportunity to integrate these distributed works. SHARE has taken a vested interest in supporting the humanities (MLA Commons is already a provider to SHARE) and this project would leverage the SHARE infrastructure to answer critical issues in DH.

The Association of Research Libraries (ARL) seeks \$75,000 in Level II funding from NEH to explore and plan, with the assistance of digital scholarship practitioners, centers, and humanities faculty, the extent to which the DH community can leverage SHARE for curation, aggregation and discovery. To complete this project, ARL will: (1) apply a mixed-methods approach to gather requirements for optimizing the SHARE aggregator and data set for digital humanities research outputs; (2) develop a strategy to harness the domain and functional knowledge of participants of the Council on Library and Information Resources (CLIR) Postdoctoral Fellows program in data curation, as well as the SHARE Curation Associates program, to enhance the SHARE data set; and (3) prototype the technical development of tools and applications that address the particular community needs of digital humanities.

Objective 1 - Gathering Requirements: Understanding requirements for an aggregated platform for digital scholarship is an integral first step. The project team will first survey 10-15 digital scholarship centers - representing a range of service models and campus owners - on metadata models, existing repositories, the types of digital assets created and archived, challenges, and workflows. Focus groups with leading humanities scholars will then work to better understand the stewardship (and gaps therein) of scholarly assets created throughout a project's lifecycle. In addition to gathering harvest

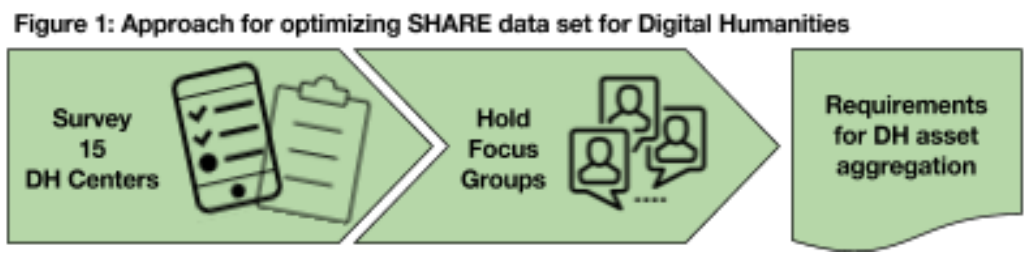
methods and aggregation requirements, focus groups will help prioritize which repositories and scholarly outputs (manuscripts, books, pre-prints, data sets, etc.) are of greatest importance for aggregation and linking. For example, by harvesting metadata about digital research tools from the DiRT directory (http://dirtdirectory.org/), the SHARE team can work with the DH community (scholars and librarians) to link these tools to research projects that have deployed them. Similarly, metadata from the registry of digital projects at DH Commons (http://dhcommons.org/projects) could, if harvested by SHARE, be linked to related intellectual works such as papers and primary sources. These exercises will also help the research library and DH communities develop a framework through which to make sound stewardship decisions for new projects.

The project team will draw upon research by Nancy L. Maron and Sarah Pickle in "Sustaining the Digital Humanities," which surfaced and analyzed faculty priorities for long-term stewardship. In the focus group component of the project, the team will engage scholars and curators in creating norms for capturing metadata reflective of discrete scholarly communication events, or moments within DH projects that would trigger a unique and granular record within the SHARE data set. A primary benefit of this component-level access to projects will be to distinguish and expose mechanisms for accessing and manipulating data from data itself. This both enables discovery and reuse of digital assets and tools for other, otherwise unrelated projects, and addresses an expressed need in the DH community to elevate tool creators in academic status on par with creators of research content.

Objective 2 - Building Community Capacity: Drawing from many repositories with highly-variable data, SHARE metadata requires enhancement and expert curation-work accomplished both through 
automatic techniques and community engagement and involvement. SHARE's Curation Associates program is a professional development and professional service opportunity for librarians to learn automated, scalable techniques of data curation. Currently in its pilot year, the program has the potential to be a community of co-learners with the CLIR Postdoctoral Fellows program in data curation, and in so doing serve as an overlapping network of expertise to further cohere data curation activities among librarians and research communities. The Associates program is partially underwritten by SHARE's current grant funding, and partially supported by the 30 libraries that are donating their participating staff time and travel money for the participants. Because collectively they represent key departments within library organizations - scholarly communication, repository management, metadata, and digital initiatives - they constitute a natural partner cohort with CLIR fellows who are new to libraries but contribute domain expertise relevant to the digital scholarship reflected in SHARE. This grant would support development of a strategy to programmatically connect these complementary initiatives.

Objective 3 - Prototyping DH Discovery: Finally, Level II funds would support two paid interns at COS to create wireframes and prototypes, responsive to community input, for open discovery platforms and tools. These prototypes will demonstrate to the DH community how aggregated metadata about highly diverse digital assets can be visualized and linked, and to see and provide feedback on the gathered requirements in development. The SHARE development team at COS is currently working with campus-based developers at UC San Diego and the University of Notre Dame on an institutional portal into the SHARE API, as well as collaborating with the Johns Hopkins University Data Conservancy's RMAP project, which is visualizing publications and data relationships using the SHARE data and API. Both of these projects demonstrate the interactive and iterative process the team envisions, and are themselves relevant to the DH community. DH scholars and librarians are keenly interested in the value DH projects contribute to institutional research, teaching and learning; and they recognize knowledge networks and relationships as worthy of critical investigation. COS has also recently launched OSF Preprints and branded disciplinary preprint services such as SocArXiv, powered by SHARE. The rapid development and uptake of SocArXiv provides a tangible example of what is possible with open data, open infrastructure, and collaboration between technology experts and disciplinary experts (Hudson et al, 2016). COS's work with similar services in psychology, engineering, agriculture, etc. to elicit common and unique features for disciplinary preprint communities can be seen on the OSF Preprints Roadmap (http://bit.ly/2iUAFGF). Applying a similarly collaborative approach, this project's resulting white paper will include a plan to enhance and extend the existing SHARE data set and infrastructure to better support the digital humanities community.

The expected benefits of funding to advance SHARE's exposure to and utility for the humanities are to: (1) bring multidisciplinary communities using common tools and practices together; (2) to increase the exposure of humanities work. Examples of digital humanities and SHARE use cases are in Appendix A, and (3) inform future development of humanities-based repositories.

\section{Environmental Scan}

Maron and Pickle (2014) analyzed the landscape of DH project production on campus from an institutional perspective-looking at service and research models, level of cross-unit coordination or lack thereof, the kinds of assets being produced, and faculty priorities for their stewardship over the long term. Preservation of digital projects and their components was an abiding concern. Ongoing sustainability was seen as dependent upon outreach efforts by project creators to maintain visibility and therefore garner ongoing technical and content support for their projects. SHARE is open infrastructure to power discovery, and can thus address these core community concerns by providing empirical data about DH projects that would enable campus units to make necessary political and financial decisions about project 
maintenance. Institutions as a whole or by unit are unlikely to standardize or enforce workflow decisions (tools, platforms, etc.) for their scholars, especially in a field as diverse as digital humanities. But knowing where projects and their components are archived, and the relationships among them, will help libraries and other campus units to optimize services and resources devoted to projects across their lifecycle. Nancy Maron will serve on this project's advisory board.

Thomas Padilla, Humanities Data Curator at the University of California Santa Barbara, will also serve on the project advisory board. As the Principal Investigator of an IMLS National Forum grant, "Always Already Computational: Library Collections as Data," (2017-18) Mr. Padilla will "produce a library collections as data framework, use cases and user stories, functional requirements for technical solutions that support library collections as data, [and] methods for making these types of collections more discoverable" (Padilla, 2016). This project, "Integrating digital humanities into the web of scholarship," will regularly interface with Padilla's IMLS project, as the aims are highly aligned and mutually informative.

To date, no other known group has planned or developed scalable technology and workflow to enhance the discovery of distributed digital humanities scholarship. The Digital Public Library of America, for example, is focused primarily on unique cultural assets or special collections and less on the scholarly outputs and full lifecycle of scholarship based on those assets and collections. The project team will investigate the existing landscape of DH registries of tools and projects, with particular attention to the Advanced Research Consortium (ARC) at Texas A\&M University, a DH aggregator of period-based online communities such as NINES and 18thConnect. Furthermore, it will explore how metadata for cultural assets aggregated through services like DPLA may complement metadata from other DH registries. As with DPLA, ARC nodes maintain their own full-text documents and digital assets but contribute metadata for central aggregation and discovery. What distinguishes SHARE's approach from DPLA and ARC is that SHARE's data is enhanced either locally by providers or in the aggregate once harvested (or both) so that participation in SHARE is maximally inclusive for lesser-resourced endeavors.

While the harvesting of metadata from distributed repositories is not unique to SHARE, no known projects have focused particularly on humanities scholarship, included such a wide variety of research outputs across the project lifecycle, or built APIs for modern data exchange and to enable new service development, as this project proposes through SHARE.

\section{History of the Project}

SHARE is a partnership between ARL, a research library member organization, and COS, a researcher-facing technology organization, that is building infrastructure to support open, web-enabled scholarly workflow in order to improve the efficiency and integrity of research. Despite its name, COS demonstrably builds tools of value to any discipline across scholarship - not just the sciences. A recent sample of approximately 1200 Open Science Framework (OSF) profiles shows active users in the humanities, social sciences, and law as well as STEM fields (Kramer and Bosman, 2016). Scholarly activity supported by the OSF and its integration with other systems, and described by SHARE, includes publications but also all forms of scholarly expression that fall outside journals and monographs. These contextual materials, important for transparency and reproducibility of scientific research, and evidentiary transparency in the humanities, are thus significant components of the 21 st century research library collection and libraries need robust access and discovery tools in order to steward them (ARL, 2012).

SHARE currently harvests metadata about research from more than 140 registries and distributed digital repositories of all kinds via an open source API that can then serve as a common API for any of these systems to exchange data with one another. With SHARE's API, scholarly activity can be easily accessed 
and linked within and among many sources to form a more complete picture of scholarship from an individual, institutional, or disciplinary perspective. SHARE is a multidisciplinary, inclusive community project open to all to participate in and use.

SHARE has been jointly and equally funded by the Alfred P. Sloan Foundation and the Institute of Museum and Library Services for \$2.2 million for 2.5 years. Over two distinct phases-first, building the pipeline and aggregator, and then, putting automated and human-curated metadata enhancement processes in place-SHARE has established itself as a necessary piece of infrastructure for linked open data about the full lifecycle of scholarly activity. With additional foundation funding, anticipated to be forthcoming from multiple sources during the proposed funding period, partners will build tools and resources on top of the SHARE data set that advance both research (e.g. meta-scholarship) and institutional objectives (stewardship).

Work plan

The Association would use requested funds from the NEH to engage digital humanities scholars and tool-builders to investigate SHARE as an aggregator to power discovery for the DH community, while at the same time pursuing greater alignment with the CLIR postdoctoral fellowship program in data curation and the SHARE Curation Associates. ARL is requesting support for 12 months from September 2017-September 2018. ARL will recruit two Visiting Program Officers (VPO) from among the existing cohort of SHARE Curation Associates so that their appointments are in place by the project start date. Using the networks of the ARL membership and the project advisory board, the project team will develop a target list of digital scholarship centers for its survey and notify them of the project and forthcoming survey during summer 2017.

Q1: September 2017-December 2017

- ARL VPOs, under the direction of the principal investigators, will:

- Develop and distribute a survey to 10-15 digital scholarship centers about workflow and stewardship of research process and objects. [See draft survey in Appendix B]

- The project team will host a workshop for SHARE representatives, CLIR representatives and library leaders to explore strategic agenda and partnership opportunities for using SHARE for humanities data curation. [See draft workshop agenda in Appendix C]

Q2: January 2018-March 2018

- Two members of the project team and one member of the COS technology staff will visit 3-4 key digital scholarship centers to hold focus groups with staff and faculty to refine and better understand their requirements for the SHARE data set. Criteria for selecting centers to visit include geographic and resource diversity, a mix of institutional homes (library or academic department), and expressed indication of willingness to spend time with the project team.

Q3: April 2018-June 2018

- Two members of the project team and one member of the COS technology staff will visit 3-4 additional key digital scholarship centers (see criteria above) to assess further the state of their technology, metadata, and workflows and engage them on participating as contributors and consumers of SHARE open data.

- ARL VPOs, with support from developers at COS, will host humanities-focused hackathons in partnership with digital scholarship centers and CLIR host universities, to build and prototype tool integration and use of SHARE open data.

Q4: July 2018-September 2018

- Project team will conduct targeted outreach to key humanities scholarly societies, including submission to conferences of the Modern Language Association and the American Historical 
Association (both of whom meet in January) to promote exposure of SHARE and its DH prototypes.

- Project team will collect feedback from other digital scholarship centers on the digital humanities prototypes that use the SHARE data set developed in Q3 through virtual user testing.

- VPOs will, under direction of the principal investigators:

- Conduct a feasibility study of moving tool prototypes into production.

- Write white paper, including technical and community outreach plan that details how the SHARE data set and tools built on top could be optimized for digital humanities scholars and digital scholarship centers.

Staff

The principal investigator, Ms. Judy Ruttenberg, and Co-principal investigator, Ms. Cynthia Hudson-Vitale, will provide general project oversight, administer the budget, be responsible for final reporting requirements, and ensure timelines and project milestones are met. The principal investigator, Ms. Ruttenberg, will manage the advisory board and recruit ARL VPOs. Ms. Hudson-Vitale will work closely with the VPOs in developing and administering surveys, recruiting focus groups participants, and connecting the CLIR fellows and SHARE Curation Associates. Staffing for this project will draw upon the strong ARL VPO program. The program provides highly visible professional development opportunities for outstanding staff members and serves the ARL membership as a whole by extending the capacity of ARL to undertake additional activities. This project will be conducted by two project leads who will join the VPO program. One will be responsible for the development of the survey and focus group instruments, workshop agenda, proceedings publication, user experience assessments, and community outreach. A second project lead will be responsible for evaluating the technical requirements for enhancing the SHARE data set for digital humanists. This individual will conduct a technical mapping of the information received from the focus groups, meetings, and survey to the SHARE data set-noting any gaps or technical development requirements. Additionally, he/she will oversee the technical feasibility study of the prototypes developed in Q3. The new VPOs will be recruited from among the existing cohort of SHARE Curation Associates, with a promising short list of candidates engaged in DH and digital scholarship work on their campuses already identified. ARL staff will provide general administrative support, including in financial reporting, communications, and event-planning. Mr. Rick Johnson, will serve in a project support role. He will work with the VPO gathering the technical requirements and serve as a conduit to the COS interns developing the prototypes. Dr. Jeffrey Spies, will travel with the VPOs to the digital scholarship centers to gather technical requirements and advise and oversee the COS interns locally in Charlottesville, VA. The project Advisory Board will help identify participating digital scholarship centers and individuals, advise on and review focus group documentation and survey instruments, and review the project's final white paper. Board members will also be invited to participate in user feedback of wireframes and prototypes.

\section{Final product and dissemination}

This project white paper will include a technical and community development plan to extend the current SHARE data set to allow for more robust digital humanities based tools and applications. A number of supporting products will be created throughout this project, including (1) the digital scholarship center survey instrument and resulting data, (2) the focus group instruments and results, (3) the workshop proceedings, (4) hackathon prototype code, (5) user experience testing results, and (6) the technical feasibility study. All products and source code created through this project will be made widely, and publically, available through the project workspace on the OSF and distributed through various channels, such as the SHARE newsletter and ARL and COS publications. 